\title{
Probiotic Strain Lactobacillus casei BL23 Prevents Colitis-Associated Colorectal Cancer
}

\author{
Elsa Jacouton', Florian Chain ${ }^{1}$, Harry Sokol ${ }^{1,2}$, Philippe Langella ${ }^{1}$ \\ and Luis G. Bermúdez-Humarán ${ }^{1 *}$
}

${ }^{1}$ Micalis Institute, INRA, AgroParisTech, Université Paris-Saclay, Jouy-en-Josas, France, ${ }^{2}$ Sorbonne Universités, UPMC Univ. Paris 06, École normale supérieure, CNRS, INSERM, APHP Laboratoire des Biomolécules (LBM), Paris, France

OPEN ACCESS

Edited by:

Jia Sun,

Jiangnan University, China

Reviewed by:

Li-Long Pan,

Fudan University, China

Jorge Gomez-Gutierrez,

University of Louisville,

United States

*Correspondence:

Luis G. Bermúdez-Humarán

luis.bermudez@inra.fr

Specialty section: This article was submitted to Nutritional Immunology,

a section of the journal

Frontiers in Immunology

Received: 12 June 2017 Accepted: 31 October 2017 Published: 17 November 2017

Citation:

Jacouton E, Chain F, Sokol $H$,

Langella $P$ and Bermúdez-

Humarán LG (2017) Probiotic Strain Lactobacillus casei BL23 Prevents Colitis-Associated Colorectal Cancer.

Front. Immunol. 8:1553. doi: 10.3389/fimmu.2017.01553
The gut microbiota plays a major role in intestinal health, and an imbalance in its composition can lead to chronic gut inflammation and a predisposition to developing colorectal cancer (CRC). Currently, the use of probiotic bacteria represents an emerging alternative to treat and prevent cancer. Moreover, consumption of these beneficial bacteria may also favorably modulate the composition of the gut microbiota, which has been described in several studies to play an important role in CRC carcinogenesis. In this context, the aim of this study was to assess the protective effect of oral treatment with Lactobacillus casei BL23, a probiotic strain well known for its anti-inflammatory and anticancer properties. First, CRC was induced in C57BL6 mice by a single intraperitoneal injection with azoxymethane $(8 \mathrm{mg} / \mathrm{kg}$ ), followed by four courses of dextran sodium sulfate $(2.5 \%)$ in drinking water that were separated by an adjustable recovery period. At the time of sacrifice (day 46), tumor incidence, histological scores, and epithelial proliferation were determined in colon samples. Our results show that $L$. casei BL23 significantly protected mice against CRC development; specifically, L. casei BL23 treatment reduced histological scores and proliferative index values. In addition, our analysis revealed that L. casei BL23 had an immunomodulatory effect, mediated through the downregulation of the IL-22 cytokine, and an antiproliferative effect, mediated through the upregulation of caspase-7, caspase-9, and Bik. Finally, L. casei BL23 treatment tended to counterbalance CRC-induced dysbiosis in mice, as demonstrated by an analysis of fecal microbiota. Altogether our results demonstrate the high potential of $L$. casei $B L 23$ for the development of new, probiotic-based strategies to fight CRC.

Keywords: Lactobacillus casei BL23, lactic acid bacteria, probiotic, azoxymethane-dextran sodium sulfate, colorectal cancer, immunomodulation

\section{INTRODUCTION}

Colorectal cancer (CRC) is a major public health problem and is considered the third most common cancer around the world, with nearly 1.2 million new cases every year and a mortality rate of $\sim 40 \%$ (1). The incidence of CRC can be associated with a large number of both genetic (2) and environmental factors (3). In particular, one major risk factor for the development of CRC is chronic intestinal inflammation (4); indeed, patients suffering from inflammatory bowel diseases (IBDs) are six times more likely to develop CRC than healthy individuals (5). 
Today, the use of probiotics represents a promising strategy for the treatment and prevention of cancer. Probiotics are "live microorganisms, which when administered in adequate amounts confer a health benefit on the host" (6). The most common probiotic strains belong to the genera Bifidobacterium and Lactobacillus. Interestingly, epidemiological studies have shown a lower incidence of CRC in healthy volunteers who regularly consumed fermented dairy products (containing probiotics), especially yogurt (7-10). However, despite encouraging observations of the anticancer effects of probiotics (which have been accumulating for over 30 years), these clues have thus far been poorly investigated, and even today, the mechanisms underlying these beneficial effects are largely unknown. The beneficial role of probiotic bacteria against CRC onset may be explained by three different mechanisms: (i) modulation of the immune response, (ii) induction of cell apoptosis, or (iii) antioxidant activity [reviewed in Ref. (11)]. As a specific example, the food supplement VSL\#3 (a mixture of eight probiotic bacteria) has been shown to modulate the immune response and reduce adenoma development in a model of CRC induced by azoxymethane (AOM) and dextran sodium sulfate (DSS) (12). Furthermore, Lactobacillus gasseri and Bifidobacterium longum have been shown to inhibit cellular proliferation and increase phagocytic activity in a model of 1,2-dimethylhydrazine (DMH)-associated CRC and thus reduce the multiplicity of aberrant crypt foci as well as tumor size (13). In addition, the probiotic strain Lactobacillus casei Shirota is able to suppress chemically induced intradermal tumor onset through both an enhancement of the cytotoxicity of natural killer (NK) cells (14) and IL-12 release by dendritic cells (15).

Beyond having cancer fighting effects, the consumption of probiotics may also favorably modulate the composition of the gut microbiota (16). In this context, several studies have confirmed the important role that the gut microbiota plays in CRC carcinogenesis by generating both biochemical and physiological conditions that may increase the number of colonic preneoplastic lesions $(17,18)$. However, despite the well-known role of microbiota dysbiosis in CRC pathogenesis, there are conflicting reports about a specific correlation between the bacterial community composition in the gut and susceptibility to CRC (18-20). To date, animal studies have revealed that germ-free mice are more predisposed to developing inflammatory-induced CRC than conventional mice (21). Therefore, manipulation of the gut microbiota could be a promising approach to prevent and/or treat CRC.

We have previously reported anti-inflammatory effects of the probiotic strain L. casei BL23 in two different murine models of chemically induced colitis (22-24). Furthermore, we have recently observed that this strain also displays antitumoral properties in a mouse allograft model of human papilloma virus (HPV)-induced cancer and in a DMH-induced CRC model (25). We attributed this antitumoral effect to a modulation of the immune response and, in particular, to the modulation of regulatory T-cells toward a Th17-biased immune response associated with the expression of regulatory cytokines (IL-6, IL-17, IL-10, and TGF- $\beta$ ) (25). However, the beneficial role of $L$. casei BL23 in colitis-associated CRC (such as that induced by AOM-DSS) remains to be assessed.
Thus, keeping in mind the role of the gut microbiota and chronic intestinal inflammation in CRC carcinogenesis, we decided in this study to further investigate the impact of L. casei BL23 in a mouse model of CRC induced by AOM and DSS. Our results showed that oral administration of L. casei BL23 significantly reduced tumor onset. Histological analyses revealed reduced proliferation in tumor sections, as demonstrated by the upregulation of three genes involved in apoptosis (caspase 7, 9, and Bik) and cytokine IL-22. Illumina sequencing revealed that the gut microbiota of mice treated with L. casei BL23 tended to differ in both community composition and community richness from those of PBS-treated mice. Finally, we provide some clues about the host molecular mechanisms involved in the anticancer effects of this beneficial probiotic bacterium.

\section{MATERIALS AND METHODS}

\section{Bacterial Strains and Growth Conditions}

Lactobacillus casei BL23 (26) was grown in Man, Rogosa, and Sharpe medium at $37^{\circ} \mathrm{C}$ without agitation overnight. To prepare live bacterial inoculum, strains were washed twice with PBS at $3,000 \mathrm{~g}$ and suspended in PBS at a concentration of $5 \times 10^{9}$ colony-forming units/ml plus $15 \%$ glycerol.

\section{Animals and Experimental Design}

All experiments were handled in accordance with institutional ethical guidelines, and the study was approved by the COMETHEA ethics committee ("Comité d'Ethique en Expérimentation Animale") of the Centre INRA of Jouy-enJosas and AgroParisTech. Female C57BL/6 mice (6-8 weeks old; Janvier SAS, St. Berthevin, France) were maintained in sterile isolators at the INRA animal facility ( $n=5$ per cage) with $12 \mathrm{~h}$ light cycles and fed irradiated normal chow (R 03-40, SAFE) and water ad libitum.

Temperature and moisture were carefully controlled. Mice were separated into three groups. The first two groups were administered PBS, while the last group was orally administered L. casei BL23. As shown in Figure 1, administration of PBS or L. casei BL23 started a week before tumor induction and was performed every day until sacrifice.

Tumors were induced with a single intraperitoneal injection of $8 \mathrm{mg} / \mathrm{kg}$ AOM (Sigma-Aldrich) 6 days before the start of a DSS treatment (with the exception of group 1, the negative control). Mice received a 5-day course of 2.5\% DSS (TdB) in sterile drinking water, followed by an adjustable recovery period. This schema was followed four times (Figure 1). Intestinal inflammation was assessed daily by measuring the Disease Activity Index (DAI), which included body weight loss $(0=<1 \%, 1=5 \%>X>1 \%$, $2=10 \%>X>5 \%, 3=15 \%>X>10 \%, 4=>15 \%)$; mouse activity $(0=$ normal, $2=$ hooked back, and $4=$ lethargy); stool consistency $(0=$ absence, $2=$ soft and sticky, and $4=$ diarrhea $)$; occult/ gross rectal bleeding $(0=$ normal, $1=$ occult,$+ 2=$ occult ++ , 3 = occult +++ , and $4=$ gross bleeding); and mouse coat state $(0=$ normal, $2=$ ruffed, and $4=$ very ruffed $)$. Mice were sacrificed at day 46. Colons were harvested and cleaned with PBS, and tumors were measured with a caliper. Colon sections were 

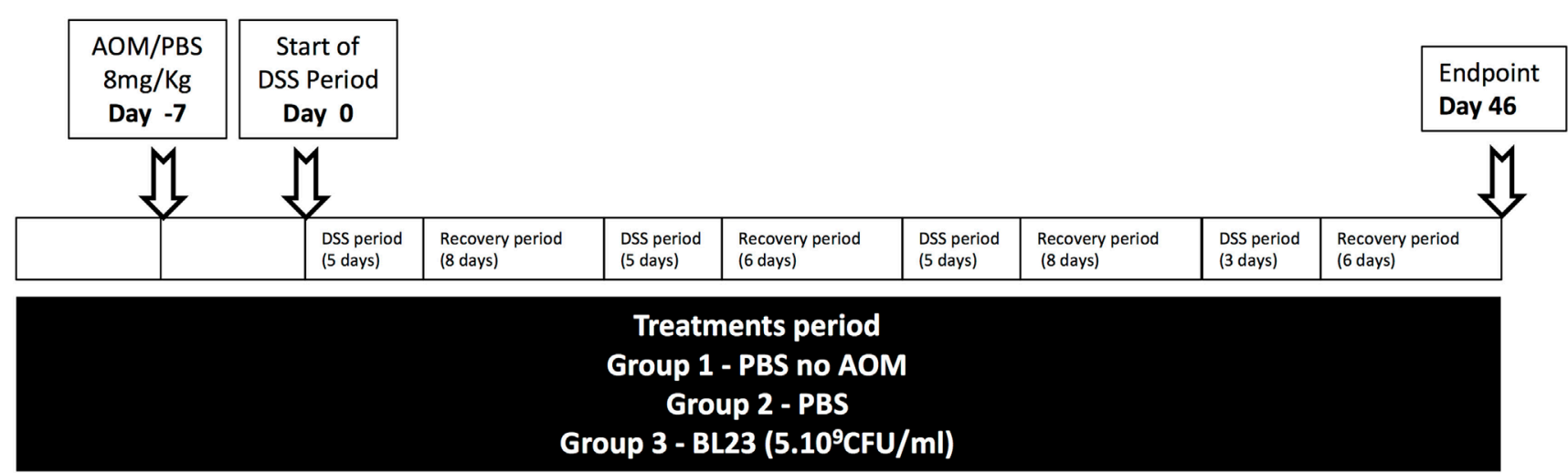

FIGURE 1 | Experimental protocol. AOM, azoxymethane; CFU, colony-forming unit; DSS, dextran sodium sulfate.

stored under conditions appropriate to the subsequent analyses (detailed in the following sections). For each mouse, three sections $(\sim 1 \mathrm{~cm})$ were recovered from rectum samples and used for histology, gene expression analysis, and protein analysis. Colic contents were frozen in liquid nitrogen.

\section{Cytokine Analysis}

Mononuclear cells were isolated from spleens by gentle extrusion of the tissue through a 50- $\mu \mathrm{m}$-mesh Nylon cell strainer (BD). Cells were resuspended in DMEM medium that was supplemented with $10 \%$ fetal calf serum, $2 \mathrm{mM}$ L-glutamine, $50 \mathrm{U} / \mathrm{mg}$ penicillin, and $50 \mathrm{U} / \mathrm{mg}$ streptomycin (Lonza, Levallois-Perret, France). Erythrocytes were lysed with red blood cell lysing buffer (Sigma-Aldrich).

For stimulation experiments, $2.5 \times 10^{6}$ cells per well were cultured for $48 \mathrm{~h}\left(37^{\circ} \mathrm{C}, 10 \% \mathrm{CO}_{2}\right)$ in DMEM medium in P24 plates that were precoated with anti-CD3/CD28 antibodies $(4 \mu \mathrm{g} / \mathrm{ml}$ each; eBioscience). Culture supernatant was frozen at $-80^{\circ} \mathrm{C}$ until processing.

Proteins from each colon were extracted with T-PER tissue protein extraction reagent (ThermoFisher Scientific) using a Fastprep instrument at $4,500 \mathrm{~g}$ for $30 \mathrm{~s}$ (two cycles). Samples were centrifuged at $500 \mathrm{~g}$ for $1 \mathrm{~min}$, and supernatants were harvested for cytokine analysis. A cytometric bead array system (LEGENDplex Mouse Th Cytokine Panel, Biolegend) was used, according to manufacturer's instructions, to determine the levels of the following cytokines: IL-2, IL-4, IL-5, IL-6, IL-9, IL-10, IL-13, IL-17A, IL-17F, IL-21, IL-22, IFN- $\gamma$, and TNF- $\alpha$.

\section{Tumor Histology}

Colon sections were formalin fixed and embedded in paraffin (4\%, VWR, France). Epithelial proliferation was assessed by Ki67 staining according to the manufacturer's instructions, using mouse monoclonal anti-Ki67 antibody (MM1, Leica Biosystems; 1:50). The proliferation index was determined by counting the number of Ki67-positive cells per crypt in three well-aligned crypts.

Two-micron colon sections were used for $\mathrm{H} \& \mathrm{E}$ staining. Histological score was determined using a BX43 Olympus microscope in a blinded manner, via the observation of three parameters: inflammation/cellular infiltration, epithelial lesions, and regeneration.

\section{Gene and Protein Expression Analysis}

Colon sections were stored in RNA later (Ambion) at $-80^{\circ} \mathrm{C}$. RNAs were extracted using the RNeasy mini-kit (Qiagen, Courtaboeuf, France) following the manufacturer's recommendations. RNA concentration was measured using a NanoDrop spectrophotometer (NanoDrop Technologies, Wilmington, DE, USA). cDNA synthesis was carried out from $1 \mu \mathrm{g}$ of RNA using the High Capacity cDNA Reverse Transcription kit (Applied Biosystems, USA), according to the manufacturer's instructions. RT-qPCR was carried out in a reaction volume of $25 \mu \mathrm{l}$ with Taqman probes ( $\beta$-actin: Mm01963702_S1, caspase-9: Mm00516563_m1, caspase-7: Mm01195085_m1, Bik: Mm00476123_m1) (Life Technologies, France) according to the manufacturer's instructions, using an ABI Prism 7700 (Applied Biosystems, USA) thermal cycler. To quantify and normalize the expression data, we used the $\Delta \mathrm{Ct}$ method, using the geometric mean $\mathrm{Ct}$ value from $\beta$-actin as the endogenous reference gene.

Total proteins were extracted from colon sections using T-PER buffer (Thermoscientific) and protease inhibitor mixture (Roche, Germany), through mechanical lysis with the Precellys homogenizer (Ozyme, France; 2 runs of 4,500 $g$ for $30 \mathrm{~s}$ ). Supernatants were collected, and western blots were performed. Briefly, samples were separated on a mini-PROTEAN TGX precast stain-free gel (4-20\%, Biorad). Blots (Trans Blot Turbo Transfer system, Biorad) were incubated with $\beta$-actin, caspase- 7 , and cleaved caspase-7 antibodies (Cell Signaling, Danvers, MA, USA).

\section{Microbial DNA Extraction}

DNA was extracted using the Godon technique from stool ( $\mathrm{T} 0=$ day of $\mathrm{AOM}$ treatment $)$ and cecal content $(\mathrm{Tf}=$ time final; day of sacrifice) as described by Lamas et al. (27). The DNA pellet was washed with $70 \%$ ethanol, dried, and resuspended in $50 \mu \mathrm{l}$ of Tris-EDTA buffer. DNA suspensions were stored at $-20^{\circ} \mathrm{C}$ until amplification. 
16S rDNA Amplification and Gene Analysis

16S rDNA was amplified with primers for the V3 and V4 hypervariable regions (PCR1F_460: 5'-CTTTCCCTACACGAC GCTCTTCCGATCTACGGRAGGCAGCAG-3', PCR1R_460:5' GGAGTTCAGACGTGTGCTCTTCCGATCTTACCAGGG TATCTAATCCT- $3^{\prime}$ ). The reaction mixture contained $10 \mathrm{ng}$ of genomic DNA, $5 \mathrm{U} / \mu \mathrm{l}$ MTP Taq DNA polymerase (Sigma, France), $0.2 \mathrm{mM}$ dNTP, and $0.5 \mu \mathrm{M}$ (final concentration) of each primer. Reactions were performed using an annealing temperature of $65^{\circ} \mathrm{C}$ for 30 cycles in a T100 thermocycler (Biorad, France). Sequencing was performed using 460-bp paired-end reads and an Illumina Miseq protocol on the GeT-PLaGe platform (Toulouse, France). Illumina reads were joined using the fastq-join method. The sequences were demultiplexed and quality filtered using the QIIME (version 1.8.0) software package. The sequences were assigned to OTUs using UCLUST algorithm 41 with a $97 \%$ threshold of pairwise identity and classified taxonomically using the Greengenes reference database.

\section{Statistical Analysis}

Data were analyzed with Prism software (version 5). All normally distributed data were displayed as mean \pm SEM. Comparisons between two groups were performed with a Student's $t$-test.

\section{RESULTS}

\section{L. casei BL23 Protects against Tumor Development in a Colitis-Associated CRC Model}

To determine the potential beneficial effects of the dairy strain BL23 of L casei on CRC onset, live bacteria were orally administered to mice treated with AOM and DSS. As shown in Figure 2A, mice fed with $L$. casei BL23 were protected against tumor development: no mouse in this group developed macroscopic tumors, compared to $67 \%$ (6/9) of mice that received only PBS (Figures 2A,B). Mice treated with PBS developed a per-mouse average of 2.3 tumors at least $2 \mathrm{~mm}$ in diameter (Figure 2C). Since this CRC model is related to chronic intestinal inflammation, we then assessed DAI and histological scores and intestinal epithelial damage. As shown in Figure 3A, DAI scores increased after each DSS cycle; however, there were no significant differences between any of the treated groups. For the histological scores, mice fed L. casei BL23 showed less damage than control mice did ( $p=0.052$, Student's $t$-test; Figures 3B,C). In addition, Ki67 levels (which are expressed in proliferating cells) were significantly lower ( $p=0.044$, Student's $t$-test) in mice treated with $L$. casei BL23 than in control mice (Figures 3D,E).

\section{L. casei BL23 Displays Antiproliferative Activities}

As immunomodulation and the induction of cell apoptosis are among the main probiotic-related protective mechanisms against CRC, we examined changes in both the immune response and apoptosis pathways due to $L$. casei BL23 treatment. We first determined the levels of both local (i.e., colon and mesenteric lymphoid node (MLN) samples) and systemic (i.e., spleen samples) cytokines that are involved in inflammation and carcinogenesis, including IL-22, IFN- $\gamma$, IL-10, IL-21, TNF- $\alpha$, IL-6, and IL-17A (Figures S1-S3 in Supplementary Material). As shown in Figure 4A, colonic IL-22 (a cytokine that promotes proliferation of cancer cells) (28) levels were lower ( $p=0.057$, Student's $t$-test) in L. casei BL23-treated mice compared to controls. Other cytokines that have been linked to CRC carcinogenesis, such as IFN- $\gamma$, TNF- $\alpha$, IL-6, IL-10, IL-21, and IL-17A, were assessed in MLN (Figure S1 in Supplementary Material), in spleen (Figure S2 in Supplementary Material), and in colon samples (Figure S3 in Supplementary Material), but no significant difference was observed between treated and control mice.

In addition, we performed colic gene expression analysis of genes involved in apoptosis, specifically, caspase-7, caspase-9, and $B i k$. Our results reveal that $L$. casei BL23 induced a significant increase compared to controls in the expression of the executioner caspase-7 (Figure 4B, $p=0.017$, Student's $t$-test) and the initiator caspase-9 (Figure 4C, $p=0.028$, Student's $t$-test), together with
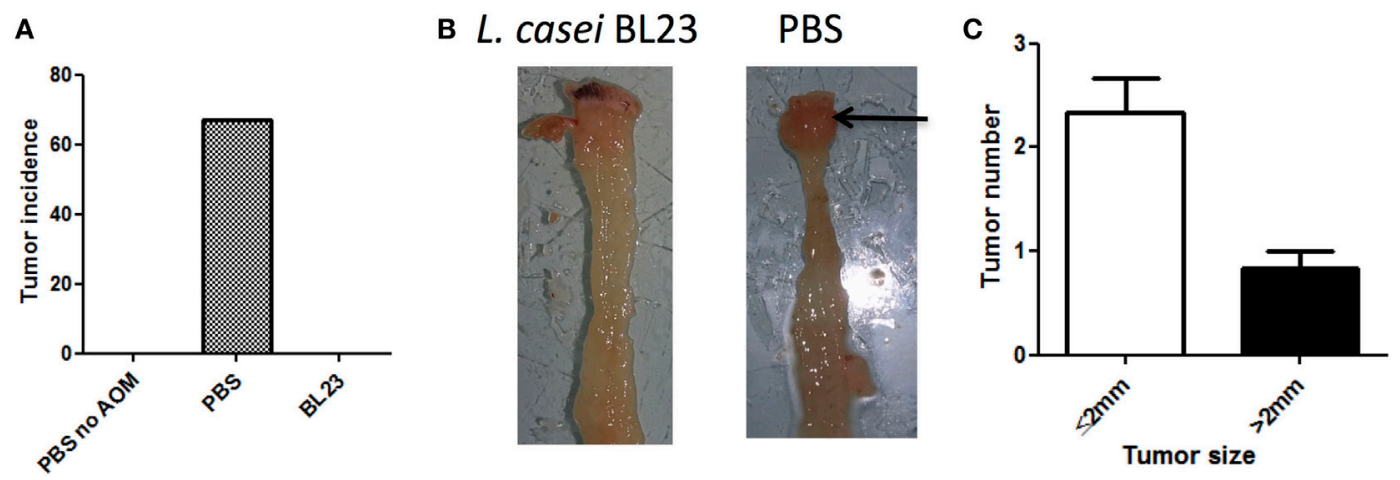

FIGURE 2 | Lactobacillus casei BL23 protected against tumor formation. (A) Macroscopic colic tumor incidence. Data are represented as the mean of each group \pm SEM ( $n=9$ mice) in an in vivo experiment. (B) Representative view of tumor in a PBS/azoxymethane (AOM)/dextran sodium sulfate (DSS)-treated mouse. (C) Colic tumor size in PBS/AOM-treated mice (number of small tumors $\leq 2 \mathrm{~mm}$ and large tumors $>2 \mathrm{~mm}$ per mouse) in the whole colon. Data are represented as the mean of each group $\pm \operatorname{SEM}(n=9$ mice). 


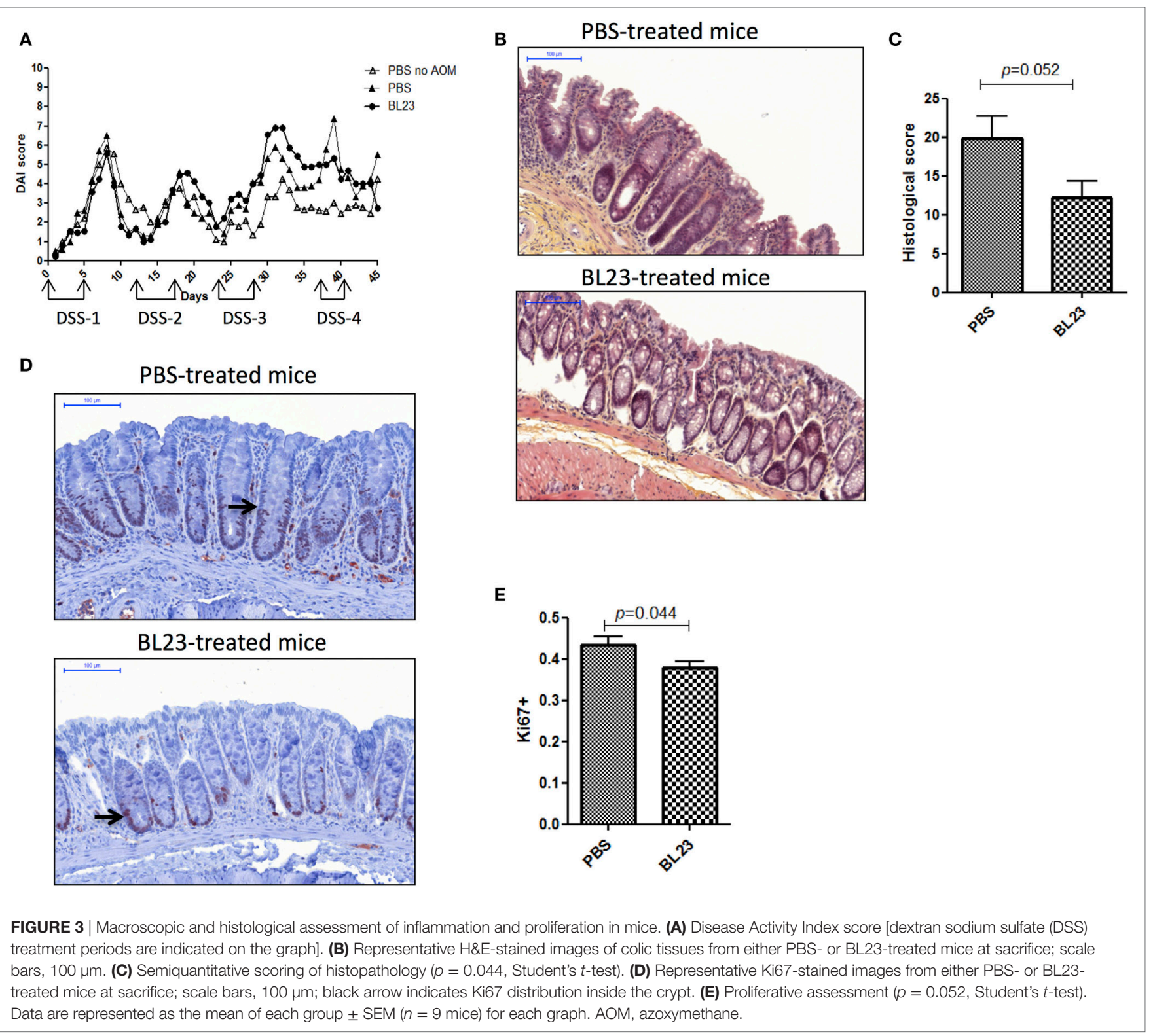

an increase of the apoptotic gene Bik (Figure 4D, $p=0.082$, Student's $t$-test). Finally, we determined the level of truncated caspase-7 (which corresponds to the active form of caspase-7 in apoptosis). For this, we selected two mice treated with $L$. case $i$ BL23 and protected against tumor development, which presented high caspase-7 RNA expression levels, and a mouse treated with PBS (not protected against tumors), which presented low levels of caspase-7 RNA expression. As shown in Figure 4E, western blot results confirmed that the mice protected against tumor onset produced higher levels of the active form of caspase- 7 compared to the non-protected mouse.

Altogether, these data suggest that L. casei BL23 has an antiproliferative and apoptotic effect in this CRC model; a detailed proposal of the mechanisms of action of L. casei BL23 against CRC is shown in Figure 5.

\section{Impact of $L$. casei BL23 Treatment on Microbiota Richness and Diversity}

To determine the impact of L. casei BL23 on the gut microbiota, we analyzed microbiota richness and diversity after BL23 oral treatment. This analysis provided a total of $1,709,762$ high-quality and classifiable reads, with an average of 5,000 $(n=51)$ reads per sample. First, beta diversity (Bray-Curtis distance) was analyzed in microbial samples using principal components analysis, which reduced the dimensionality of the data set. While no grouping was observed before AOM challenge (T0; Figure 6A), the microbiota of mice treated with $L$. casei BL23 tended to diverge from those of PBS-treated animals at Tf (sacrifice; Figure 6B, $p=0.1$, Anosim). Then, we estimated community richness (alpha diversity; Shannon and Simpson indexes) at T0 and Tf. As shown in Figure 6C, AOM injection resulted in a reduction 

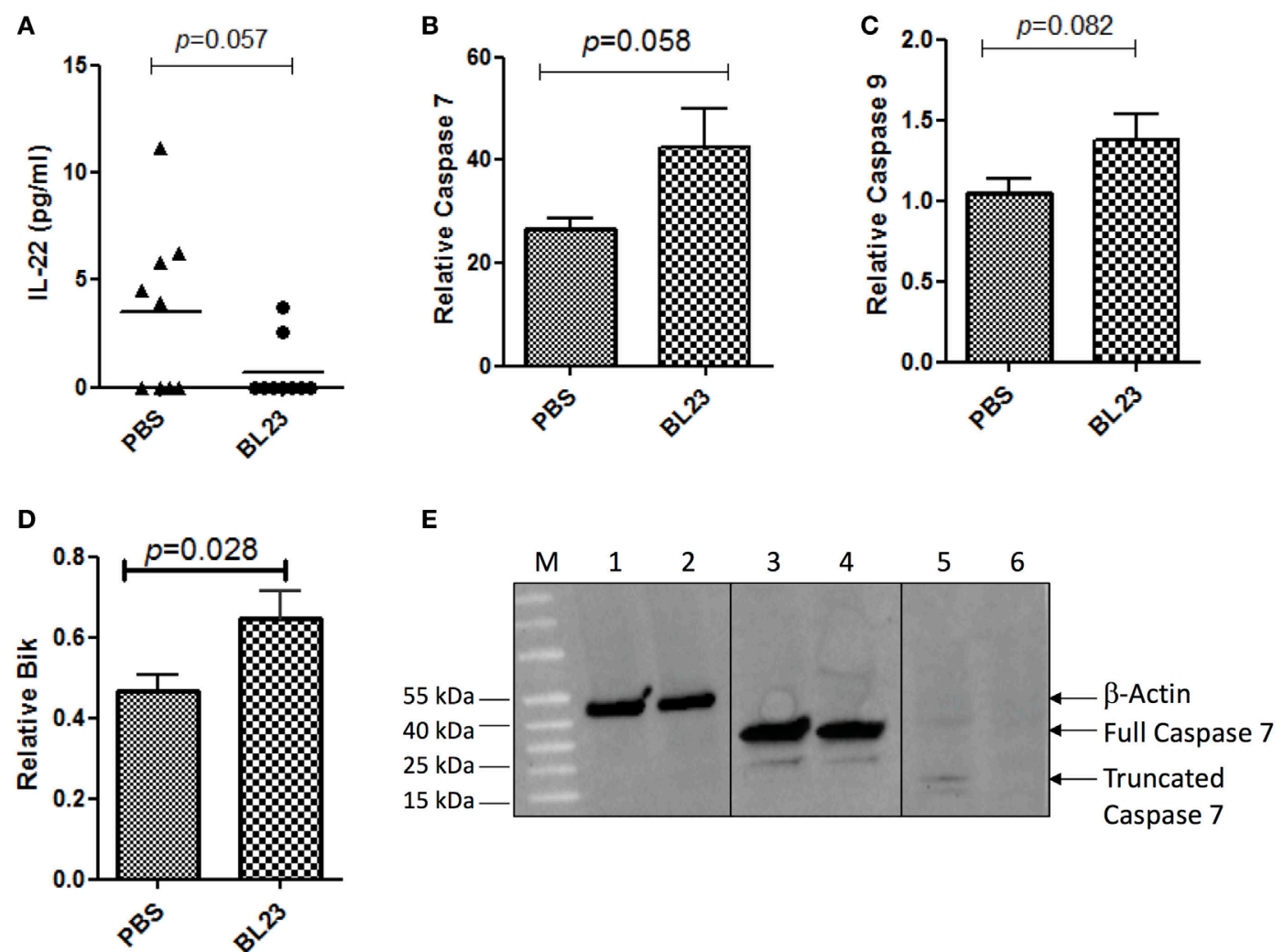

FIGURE 4 | Lactobacillus casei BL23 induced antiproliferative activity and increased apoptosis. (A) Protein analysis of IL-22 in colon tumor section. (B) Real-time PCR analysis of relative expression in colic tumor sections of mRNA of caspase-7 ( $p=0.058$, Student's $t$-test), (C) caspase- 9 ( $p=0.082$, Student's $t$-test), and (D) Bik ( $p=0.028$, Student's $t$-test). Data are represented as the mean \pm SEM of each group $(n=9$ mice) for each graph. (E) Caspase 7 protein expression. Lines 1,3 , and 5 correspond to mice treated with L. casei BL23 (and protected against tumors and that expressed high levels of caspase-7 RNA) and lines 2 , 4 , and 6 to mice treated with PBS (not protected against tumors). Lines 1 and 2 correspond to samples treated with anti- $\beta$-actin antibodies; 3 and 4 with full caspase 7 antibodies; and 5 and 6 with antibodies recognizing the cleaved form of caspase 7.

in alpha diversity with respect to that found in the group treated with PBS but not AOM (Shannon index, $6.4 \pm 0.22$ in PBS/ AOM-treated group versus $6.8 \pm 0.46$ in $\mathrm{PBS} /$ no AOM group, $p=0.028$, Student's $t$-test). Despite the fact that the effects of L. casei strain BL23 were not statistically significant (Simpson index, $483.3 \pm 111.8$ in BL23/AOM-treated group versus $436.8 \pm 54.7$ in PBS/AOM-treated group, ns, Student's $t$-test; Figure 6D), these results provide intriguing clues about this strain's interactions with other members of the gut microbiota.

Finally, bacterial communities were characterized at the phylum level (Figure 6E). Firmicutes was the dominant phylum in the L. casei BL23/AOM treated group ( $51 \pm 10 \%)$, with Bacteroidetes ranked second $(45 \pm 10 \%)$. In contrast, Bacteroidetes was the most abundant phylum in both PBS/AOM and PBS/no AOM treated groups ( $52 \pm 12$ and $53 \pm 13 \%$, respectively), followed by Firmicutes ( $44 \pm 12$ and $43 \pm 13 \%$, respectively). The third most abundant phylum in all groups was Proteobacteria, with approximately $3-4 \%$ of reads. By using linear discriminant analysis (LDA) coupled with effect size measurements (linear discriminant analysis effect size), we found that Prevotella, Ruminococcaceae, and Lactobacillus were the key groups that were overrepresented in the L. casei BL23-treated mice (Table 1). At the species level, only Lactobacillus zeae was significantly more abundant ( $p=0.004$, Student's $t$-test) in these mice, with a LDA score of 2.58. This was not surprising, since this species could in fact correspond to strain BL23 of L. casei. Indeed, 16S RNA analysis has revealed a close relationship (99\% similarity) between our focal strain and L. zeae (26).

\section{DISCUSSION}

There is now mounting evidence pointing to an important link between both chronic colic and rectal damage (present, for example, in IBD patients) and CRC carcinogenesis. Indeed, the risk of developing colitis-associated cancer increases by $\sim 1 \%$ in IBD patients (5). To better understand the mechanisms related to tumor onset, different preclinical animal models of CRC have been developed, such as the AOM-DSS model used in this study (29).

L. casei BL23 has been previously studied for its anti-inflammatory activities in different models of chemically induced colitis $(22,23)$. Furthermore, L. casei has been widely studied in different murine models of cancer $(14,30-32)$. In particular, L. casei BL23 has antiproliferative effects in the mouse allograft model of $\mathrm{HPV}$-induced cancer and protects against DMH-induced CRC (25). Here, we decided to explore the impact of oral administration of L. casei strain BL23 in a murine model of CRC induced by 


\section{L. casei BL23 improves colitis associated colorectal cancer}

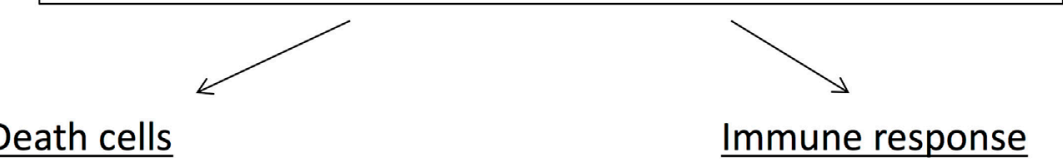

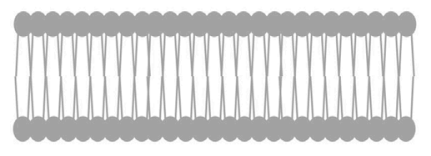

Intrinsic pathway

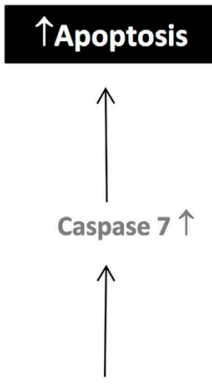

Caspase $9 \uparrow$

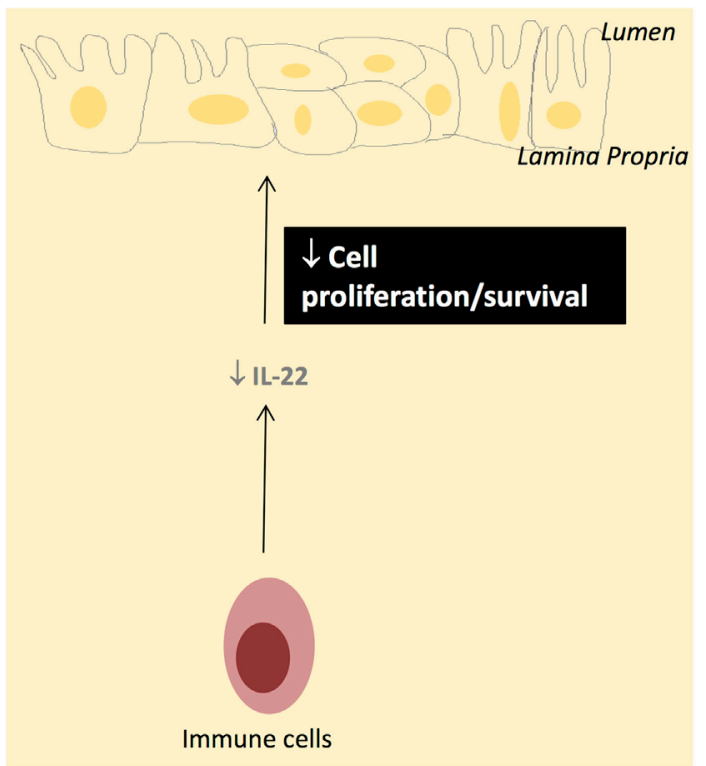

FIGURE 5 | Proposed mechanisms by which Lactobacillus casei BL23 protects against tumors.

AOM and DSS. Strikingly, our results revealed that L. casei BL23 significantly reduced tumor development, since all treated mice were tumor free at the end of the experiment. In addition, we also found some clues about the molecular mechanisms involved in the protective effect against cancer, and it appears that, in this model, L. casei BL23 acts mainly via the inhibition of cell proliferation. Indeed, this strain is able to downregulate proliferation, as observed through a decrease in Ki67. In addition, L. casei BL23 was also able to increase apoptosis via upregulation of caspase-9, caspase-7, and Bik.

Given the reported anti-inflammatory properties of $L$. casei BL23, we also assessed the cytokine profiles of treated mice. With the exception of a reduced histological score, L. casei BL23 had no significant effects on cytokine regulation. However, a weak decrease was observed in IL-22 levels in colons from mice treated with BL23. This cytokine has been recently implicated in CRC development in both humans and $\mathrm{APC}^{\mathrm{min} /+}$ murine model (33). Thus, it appears that IL-22 levels are enhanced in tumor tissues and that mice displaying lower levels of this cytokine are protected from tumorigenesis. It was recently reported that the beneficial effects of a strain of Lactobacillus reuteri in a model of CRC induced by AOM-DSS were mediated by a histidine decarboxylase (HDC), which downregulated IL-22 expression (34). We performed in silico analyses to search for the nucleotide sequence of the HDC cluster in the L. casei BL23 genome, but were unable to find a corresponding sequence region (data not shown). However, it is still possible that another protein produced by this strain may act directly on IL-22 regulation/expression. The main sources of IL-22 are NK cells, $\gamma \delta$ T cells, and lymphoid tissue inducer cells, as well as TH17 and TH22 cells. To determine which cell types are affected by $L$. casei BL23 treatment, future experiments will need to examine the correlation between each population of cells in the lamina propria of mice and IL-22 downregulation.

Finally, several reports have described disruption of the composition of the microbiota in CRC $(17,18)$. Therefore, we analyzed the bacterial diversity (16S rDNA) in fecal samples from our mice and found that L. casei BL23 tended to restore the diversity disrupted by AOM injection. In agreement with previous reports (20), Firmicutes was the dominant phylum in the AOM/PBS-treated group. However, the introduction of $L$. casei BL23 reversed the ratio of Firmicutes to Bacteroidetes. Few individual species were affected by $L$. casei treatment, with the exception of L. zeae, which is actually considered a synonym of L. casei strain BL23 (26). However, future investigations should also consider analyzing the bacterial communities in the colic mucosa from tumor sections, because the assemblages present in stools are not necessarily an accurate reflection of the intestinal environment.

In conclusion, our work revealed that L. casei strain BL23 protected against CRC in an AOM/DSS model. Although this bacterium has well-known anti-inflammatory properties, we speculate instead that the protection observed here occurs through a reduction in cell proliferation and the induction of apoptosis. 


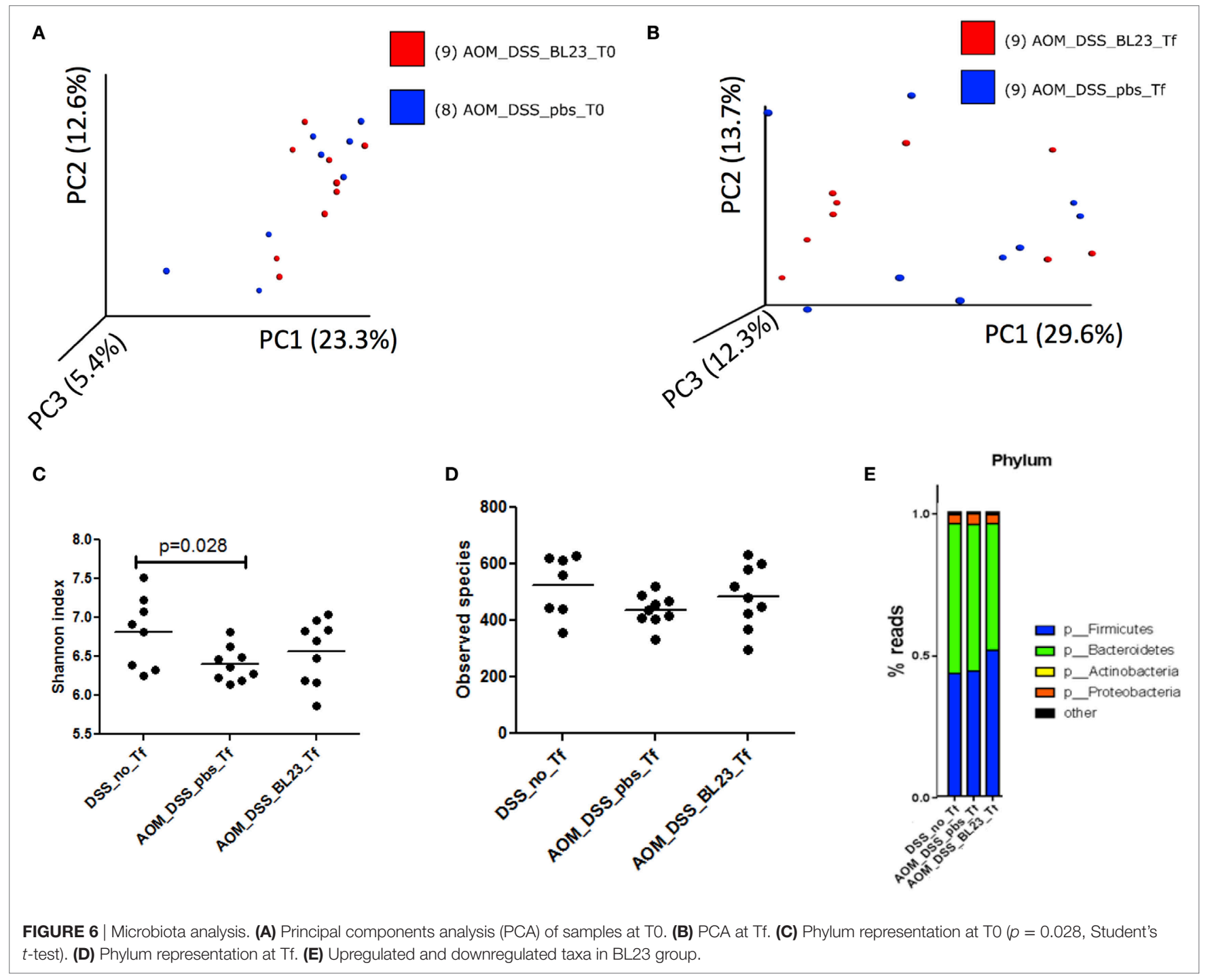

TABLE 1 | Linear discriminant analysis effect size in BL23-treated group at Tf.

\begin{tabular}{|c|c|c|}
\hline Taxa & LDA score & $p$ Value \\
\hline $\begin{array}{l}\text { Bacteroidetes. Bacteroidia. Bacteroidales. } \\
\text { Paraprevotellaceae. Prevotella }\end{array}$ & 3.2891683 & 0.038 \\
\hline $\begin{array}{l}\text { Firmicutes. Clostridia. Clostridiales. } \\
\text { Ruminococcaceae }\end{array}$ & 3.01945662 & 0.012 \\
\hline $\begin{array}{l}\text { Firmicutes. Bacilli. Lactobacillales. } \\
\text { Lactobacillaceae. Lactobacillus zeae }\end{array}$ & 2.58044456 & 0.004 \\
\hline Tenericutes & 2.573562 & 0.040 \\
\hline Tenericutes. Mollicutes & 2.57347147 & 0.040 \\
\hline $\begin{array}{l}\text { Actinobacteria. Actinobacteria. Bifidobacteriales. } \\
\text { Bifidobarteriaceae. Bifidobacterium }\end{array}$ & -0.44318815 & 0.004 \\
\hline Firmicutes. Clostridia. Clostridiales & -0.43837615 & 0.011 \\
\hline Actinobacteria. Actinobacteria & -0.42251683 & 0.018 \\
\hline $\begin{array}{l}\text { Actinobacteria. Actinobacteria. Bifidobacteriales. } \\
\text { Bifidobacteriaceae. Bifidobacterium }\end{array}$ & -0.40710059 & 0.029 \\
\hline $\begin{array}{l}\text { Firmicutes. Clostridia. Clostridiales. } \\
\text { Clostridiaceae. Clostridium }\end{array}$ & -0.38186026 & 0.030 \\
\hline Proteobacteria. Alphaproteobacteria & -0.367064 & 0.019 \\
\hline
\end{tabular}

\section{ETHICS STATEMENT}

All experiments were handled in accordance with institutional ethical guidelines, and the study was approved by the COMETHEA ethics committee ("Comité d'Ethique en Expérimentation Animale") of the Centre INRA of Jouy-enJosas and AgroParisTech. Female C57BL/6 mice (6-8 weeks old; Janvier SAS, St. Berthevin, France) were maintained in sterile isolators at the INRA animal facility ( $n=5$ per cage) with $12 \mathrm{~h}$ light cycles and fed irradiated normal chow (R 03-40, SAFE) and water ad libitum.

\section{AUTHOR CONTRIBUTIONS}

EJ, LB-H, and FC conceived and designed the study. EJ and HS performed data analysis. EJ and LB-H wrote the manuscript. EJ conducted all experiments. FC provided technical help for the in vivo experiments. EJ, LB-H, PL, and HS discussed the experiments and results. 


\section{ACKNOWLEDGMENTS}

The authors thank the members of the animal facility of INRA and the histology facility of UMR 1313 GABI, Jouy-en-Josas, France for their technical help. They also thank the MIMA2 platform for access to the virtual slide scanner (Pannoramic SCAN, 3DHISTECH).

\section{FUNDING}

This work was partially funded by the Association pour la Recherche sur le Cancer (ARC, France): action no. PGA120140 20851.

\section{REFERENCES}

1. Ferlay J, Soerjomataram I, Dikshit R, Eser S, Mathers C, Rebelo M, et al. Cancer incidence and mortality worldwide: sources, methods and major patterns in GLOBOCAN 2012. Int J Cancer (2015) 136:E359-86. doi:10.1002/ ijc. 29210

2. Muller MF, Ibrahim AE, Arends MJ. Molecular pathological classification of colorectal cancer. Virchows Arch (2016) 469:125-34. doi:10.1007/s00428016-1956-3

3. Bishehsari F, Mahdavinia M, Vacca M, Malekzadeh R, Mariani-Costantini R. Epidemiological transition of colorectal cancer in developing countries: environmental factors, molecular pathways, and opportunities for prevention. World J Gastroenterol (2014) 20:6055-72. doi:10.3748/wjg.v20.i20.6055

4. Izano M, Wei EK, Tai C, Swede H, Gregorich S, Harris TB, et al. Chronic inflammation and risk of colorectal and other obesity-related cancers: the health, aging and body composition study. Int J Cancer (2016) 138:1118-28. doi:10.1002/ijc.29868

5. Mattar MC, Lough D, Pishvaian MJ, Charabaty A. Current management of inflammatory bowel disease and colorectal cancer. Gastrointest Cancer Res (2011) 4:53-61.

6. Food and Agriculture Organization of the United Nations. Joint FAO/WHO Working Group Report on Drafting Guidelines for the Evaluation of Probiotics in Food. London: Food and Agriculture Organization (2002). 11 p.

7. Malhotra SL. Dietary factors in a study of cancer colon from Cancer Registry, with special reference to the role of saliva, milk and fermented milk products and vegetable fibre. Med Hypotheses (1977) 3:122-6. doi:10.1016/03069877(77)90024-X

8. Young TB, Wolf DA. Case-control study of proximal and distal colon cancer and diet in Wisconsin. Int J Cancer (1988) 42:167-75. doi:10.1002/ ijc.2910420205

9. Peters RK, Pike MC, Garabrant D, Mack TM. Diet and colon cancer in Los Angeles County, California. Cancer Causes Control (1992) 3:457-73. doi:10.1007/BF00051359

10. Kampman E, Goldbohm RA, Van Den Brandt PA, Van 'T Veer P. Fermented dairy products, calcium, and colorectal cancer in The Netherlands Cohort Study. Cancer Res (1994) 54:3186-90.

11. Zhong L, Zhang X, Covasa M. Emerging roles of lactic acid bacteria in protection against colorectal cancer. World J Gastroenterol (2014) 20:7878-86. doi:10.3748/wjg.v20.i24.7878

12. Bassaganya-Riera J, Viladomiu M, Pedragosa M, De Simone C, Hontecillas R. Immunoregulatory mechanisms underlying prevention of colitis-associated colorectal cancer by probiotic bacteria. PLoS One (2012) 7:e34676. doi:10.1371/ journal.pone.0034676

13. Foo NP, Yang HO, Chiu HH, Chan HY, Liao CC, Yu CK, et al. Probiotics prevent the development of 1,2-dimethylhydrazine (DMH)-induced colonic tumorigenesis through suppressed colonic mucosa cellular proliferation and increased stimulation of macrophages. J Agric Food Chem (2011) 59:13337-45. doi:10.1021/jf203444d

14. Takagi A, Matsuzaki T, Sato M, Nomoto K, Morotomi M, Yokokura T. Enhancement of natural killer cytotoxicity delayed murine carcinogenesis by a probiotic microorganism. Carcinogenesis (2001) 22:599-605. doi:10.1093/ carcin/22.4.599

\section{SUPPLEMENTARY MATERIAL}

The Supplementary Material for this article can be found online at http://www.frontiersin.org/article/10.3389/fimmu.2017.01553/ full\#supplementary-material.

FIGURE S1 | Cytokine expression levels in mesenteric lymphoid node. (A) IFN- $\gamma$, (B) IL-17A, (C) IL-6, (D) TNF- $\alpha$, (E) IL-10, and (F) IL-22. Medians are represented for each group.

FIGURE S2 | Cytokine expression levels in spleen. (A) IFN- $\gamma$, (B) IL-17A, (C) IL-6, (D) IL-22, (E) TNF- $\alpha$, (F) IL-10, and (G) IL-21. Medians are represented for each group.

FIGURE S3 | Cytokine expression levels in colon. (A) IFN- $\gamma$, (B) IL-17A, (C) IL-6, (D) TNF- $\alpha$, (E) IL-10, and (F) IL-21. Medians are represented for each group.

15. Takagi A, Ikemura H, Matsuzaki T, Sato M, Nomoto K, Morotomi M, et al. Relationship between the in vitro response of dendritic cells to Lactobacillus and prevention of tumorigenesis in the mouse. JGastroenterol (2008) 43:661-9. doi:10.1007/s00535-008-2212-7

16. He M, Shi B. Gut microbiota as a potential target of metabolic syndrome: the role of probiotics and prebiotics. Cell Biosci (2017) 7:54. doi:10.1186/ s13578-017-0183-1

17. Uronis JM, Muhlbauer M, Herfarth HH, Rubinas TC, Jones GS, Jobin C. Modulation of the intestinal microbiota alters colitis-associated colorectal cancer susceptibility. PLoS One (2009) 4:e6026. doi:10.1371/journal.pone. 0006026

18. Sobhani I, Tap J, Roudot-Thoraval F, Roperch JP, Letulle S, Langella P, et al. Microbial dysbiosis in colorectal cancer (CRC) patients. PLoS One (2011) 6:e16393. doi:10.1371/journal.pone.0016393

19. Marchesi JR, Dutilh BE, Hall N, Peters WH, Roelofs R, Boleij A, et al. Towards the human colorectal cancer microbiome. PLoS One (2011) 6:e20447. doi:10.1371/journal.pone.0020447

20. Gao ZG, Guo BM, Gao RY, Zhu QC, Qin HL. Microbiota dysbiosis is associated with colorectal cancer. Front Microbiol (2015) 6:20. doi:10.3389/ fmicb.2015.00020

21. Zhan Y, Chen PJ, Sadler WD, Wang FY, Poe S, Nunez G, et al. Gut microbiota protects against gastrointestinal tumorigenesis caused by epithelial injury. Cancer Res (2013) 73:7199-210. doi:10.1158/0008-5472.CAN-13-0827

22. Rochat T, Bermudez-Humaran L, Gratadoux JJ, Fourage C, Hoebler C, Corthier G, et al. Anti-inflammatory effects of Lactobacillus casei BL23 producing or not a manganese-dependant catalase on DSS-induced colitis in mice. Microb Cell Fact (2007) 6:22. doi:10.1186/1475-2859-6-22

23. Watterlot L, Rochat T, Sokol H, Cherbuy C, Bouloufa I, Lefevre F, et al. Intragastric administration of a superoxide dismutase-producing recombinant Lactobacillus casei BL23 strain attenuates DSS colitis in mice. Int J Food Microbiol (2010) 144:35-41. doi:10.1016/j.ijfoodmicro.2010. 03.037

24. Leblanc JG, Del Carmen S, Miyoshi A, Azevedo V, Sesma F, Langella P, et al. Use of superoxide dismutase and catalase producing lactic acid bacteria in TNBS induced Crohn's disease in mice. J Biotechnol (2011) 151:287-93. doi:10.1016/j.jbiotec.2010.11.008

25. Lenoir M, Del Carmen S, Cortes-Perez NG, Lozano-Ojalvo D, MunozProvencio D, Chain F, et al. Lactobacillus casei BL23 regulates Treg and Th17 T-cell populations and reduces $\mathrm{DMH}$-associated colorectal cancer. J Gastroenterol (2016) 51(9):862-73. doi:10.1007/s00535-0151158-9

26. Acedo-Felix E, Perez-Martinez G. Significant differences between Lactobacillus casei subsp casei ATCC 393(T) and a commonly used plasmid-cured derivative revealed by a polyphasic study. Int J Syst Evol Microbiol (2003) 53:67-75. doi:10.1099/ijs.0.02325-0

27. Lamas B, Richard ML, Leducq V, Pham HP, Michel ML, Da Costa G, et al. CARD9 impacts colitis by altering gut microbiota metabolism of tryptophan into aryl hydrocarbon receptor ligands. Nat Med (2016) 22:598. doi:10.1038/ nm.4102

28. Perusina Lanfranca M, Lin Y, Fang J, Zou W, Frankel T. Biological and pathological activities of interleukin-22. J Mol Med (Berl) (2016) 94:523-34. doi:10.1007/s00109-016-1391-6 
29. Tanaka T, Kohno H, Suzuki R, Yamada Y, Sugie S, Mori H. A novel inflammation-related mouse colon carcinogenesis model induced by azoxymethane and dextran sodium sulfate. Cancer Sci (2003) 94:965-73. doi:10.1111/j.13497006.2003.tb01386.x

30. Lee JW, Shin JG, Kim EH, Kang HE, Yim IB, Kim JY, et al. Immunomodulatory and antitumor effects in vivo by the cytoplasmic fraction of Lactobacillus casei and Bifidobacterium longum. J Vet Sci (2004) 5:41-8.

31. Kumar A, Singh NK, Sinha PR. Inhibition of 1,2-dimethylhydrazine induced colon genotoxicity in rats by the administration of probiotic curd. Mol Biol Rep (2010) 37:1373-6. doi:10.1007/s11033-009-9519-1

32. Kumar A, Singh NK, Sinha PR, Kumar R. Intervention of Acidophilus-casei dahi and wheat bran against molecular alteration in colon carcinogenesis. Mol Biol Rep (2010) 37:621-7. doi:10.1007/s11033-009-9649-5

33. Huber S, Gagliani N, Zenewicz LA, Huber FJ, Bosurgi L, Hu B, et al. IL-22BP is regulated by the inflammasome and modulates tumorigenesis in the intestine. Nature (2012) 491:259-63. doi:10.1038/nature11535
34. Gao C, Ganesh BP, Shi Z, Shah RR, Fultz R, Major A, et al. Gut microbemediated suppression of inflammation-associated colon carcinogenesis by luminal histamine production. Am J Pathol (2017) 187:2323-36. doi:10.1016/ j.ajpath.2017.06.011

Conflict of Interest Statement: The authors declare that the research was conducted in the absence of any commercial or financial relationships that could be construed as a potential conflict of interest.

Copyright (c) 2017 Jacouton, Chain, Sokol, Langella and Bermúdez-Humarán. This is an open-access article distributed under the terms of the Creative Commons Attribution License (CC BY). The use, distribution or reproduction in other forums is permitted, provided the original author(s) or licensor are credited and that the original publication in this journal is cited, in accordance with accepted academic practice. No use, distribution or reproduction is permitted which does not comply with these terms. 